\title{
Macro- and microscopic spectral-polarization characteristics of the structure of normal and abnormally located chordae tendianeae of left ventricular
}

\author{
Yu.Yu. Malyk ${ }^{\mathrm{a}}$, O.G. Prydij*b ${ }^{\mathrm{b}}$, D.A.Zymnyakov ${ }^{\mathrm{c}}$, M.V.Alonova $^{\mathrm{c}}$, O.V.Ushakova ${ }^{\mathrm{c}}$ \\ ${ }^{a}$ Bukovinian State Medical University, 2 Theatre Sq., Chernivtsi, 58000, Ukraine \\ ${ }^{\mathrm{b}}$ Chernivtsi National University, 2 Kotsyubinsky Str., Chernivtsi, 58012, Ukraine \\ 'Saratov State Technical University, Polytechnicheskaya st., 77, Saratov, 410054, Russia
}

\begin{abstract}
The morphological peculiarities of TS mitral valve of the heart of man in normal and abnormal spaced strings of the left ventricle and the study of their structural features depending on the location was studied. There are given the results of comparative statistics, correlation and fractal study population Mueller-matrix images (MMI) of healthy and abnormal (early forms that are not diagnosed by histological methods) BT normal and abnormally located tendon strings left ventricle of the human heart. Abnormalities in the structure of the wings, tendon strings (TS), mastoid muscle (MM) in inconsistencies elements and harmonized operation of all valve complex shown in the features of the polarization manifestations of it laser images.
\end{abstract}

Keywords: strings, chordae tendianeae, polarization images, biological tissue, autocorrelation function

\section{INTRODUCTION}

It is difficult to overestimate the role of valvular heart in the functioning of the cardiovascular system as well as in the development of various pathological processes structures and cardiovascular disorders ${ }^{1}$.

Normal functioning of the valve complex depends primarily on the relationship of its structural components. Abnormalities in the structure of the wings, tendon strings (TS), mastoid muscle (MM) leads to inconsistencies elements and harmonized operation of all valve complex.

The problem of the syndrome connective tissue dysplasia ${ }^{2}$ (CTD) has attracted researchers' attention because of the high incidence, and because of the risk of serious complications. From a clinical point of view , the most important reason cardiovascular manifestations of CTD, because they represent a life-threatening factors ${ }^{2}$. The most common manifestations of CTD cardiovascular system include the heart valve prolapse and abnormal are strings (APC). These states are defined as small anomalies of the heart (MAC) characterized by persistent anatomical changes that lead to the "weakness" connective tissue "skeleton" of the organ, but not accompanied by clinically and hemodynamically significant abnormalities. The fact that the mass of the population are quite common and many people do not manifest clinical abnormalities, allows some students to consider their options for normal or border states. However, these small abnormalities can cause over the years to become a wide variety of complications and other pathological conditions and diseases $^{1-2}$ and the cause of premature mortality. Therefore, distinguishing MAC from numerous variations of the rules is very difficult and is a key issue for further detailed study of them, and the increasing incidence of cardiovascular profile makes high demands on developing effective methods of diagnosis and correction of various pathological conditions of the heart.

The modern methods of investigation of the structure of biological tissues (BT) are laser polarimetry using different methods of analysis of the data, which are based on such fundamental concepts as the polarization properties of light that describes the coherence matrix ${ }^{3-8}$.

Today, generally considered the following approach for modeling the properties of biological tissues: the diversity of BT person can be represented as four main types (connective, muscle, epithelial and nervous), morphological structure which is a two-component amorphous-crystalline (isotropic and anisotropic optical components) structure ${ }^{9-12}$. Opticalanisotropic component consists of two levels of the organization - crystalline and architectonic. Crystalline component

*yeserg@rambler.ru; phone/fax +30372244730

Eleventh International Conference on Correlation Optics, edited by Oleg V. Angelsky, Proc. of SPIE Vol. 9066, 90661Q · C 2013 SPIE · CCC code: 0277-786X/13/\$18 · doi: 10.1117/12.2053541 
is the architectonic snare, consisting of protein (collagen, elastic, etc.). Fibrils that possess properties uniaxial liquid crystals ${ }^{5}$. However, these parameters of the structure can not be detected by light or electron microscopy. The above crystalline properties are good because of their ability to change the plane of polarization of the light beam. Photometric, spectral, polarization and correlation characteristics of the field scattered by biological layers of radiation become carriers of information about their properties ${ }^{6-8}$. Since the foundation SS is the connective tissue, the main components of which are collagen and elastic fibers, and does not rule out the location of the muscle cells, it would be worthwhile to study the polarization characteristics of their images. These laser images of histological sections complete the results of research about the features of connective tissue ultrastructure SS mitral valve in normal people as well as their abnormal location. This knowledge will help in the prediction and prevention of complications such as rupture strings arrhythmic syndrome, thrombosis of vessels and others. Arising in individuals with abnormally spaced strings. The knowledge of the rules in the future will be the basis for the differential diagnosis of diseases, including valvular heart disease.

The purpose of our study was to investigate the morphological peculiarities of TS mitral valve of the heart of man in normal and abnormal spaced strings (ASS) of the left ventricle and the study of their structural features depending on the location.

\section{MICRO- AND MACROSCOPIC DESCRIPTION OF TENDOR STRINGS}

TS are components of valvular heart - thin fibrous bands that come in the form of threads from the edges of leaves to the tops SM or trabeculae, blood supply and innervation which occurs through the top of SM. In the course they can be divided into multiple threads ${ }^{1-2}$. According to the classification of SS they are divided into territorial, that is, those that are attached to the edges of the leaves, leaf, place attachment which is the lower leaf surface of the valve. Strings that are not attached to the cusps are defined as abnormally located. Topography and structure of the SS affects the normal functioning of valvular heart disease and its hemodynamics, and therefore causes an increased interest in their structural organization in normal and pathological conditions.

At macroscopic examination TS mitral valve heart they stretched from the top front of MM, sometimes on their upper third, the front wing valve, and on the way it consistently (1-4 times) separated strings on the first, second and third order. TS that attached to the wings vary. To the free edge of the front doors 10 to 20 strings were connected, the ventricular surface suited for 7-15 strings. Posterior mitral valve is also attached to the TS of 20-50 to MM. For the free edge fixed rear wing 20-45 strings to the ventricular surface - 10-20 strings.

TS from each MM attached to the corresponding leaf and to a lesser extent, to the next. The strings were recorded not only on the free edge, but also to ventricular surface cusps and annulus fibrosus.

A study of light optical sections arranged TS normal mitral valve stained with hematoxylin-eosin for an overview, as well as by Veyherta Van and Van Hizona Hizonom to visualize collagen and elastic fibers and differentiate them from the muscle cells. We have found the following morphological features of the microscopic structure: the surface normally located all TS was covered with endocardium, consisting of endothelial surface layer and deeper layer situated elastic fibers formed a loose net. TS skeleton was dense connective tissue, such as tightly packed, sent straight wavy bundles of collagen and less elastic fibers interwoven (Fig. 1).

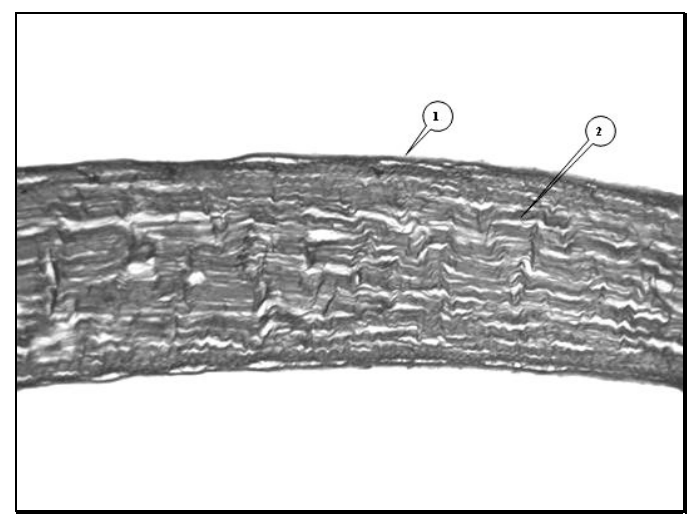

Figure 1. Longitudinal section normally located tendon strings mitral valve. $\times 40$. Staining method Veyherta Van Hizona. 1 - endothelium, 2 - bundles of collagen fibers 
The dynamics of change histostructure tendon strings can also be traced in the study sites of attachment, which is accumulation of muscle tissue, and the presence of blood vessels, layers of loose connective tissue. Histological study area discharge of tendon strings of mastoid muscle revealed that collagen fiber mop tops in MM look originally, most clearly seen in sections stained by van Hizonu (Fig. 2). And cardiomyocytes are intertwined with each other get into a string, usually a short distance, often accompanied by blood vessels.

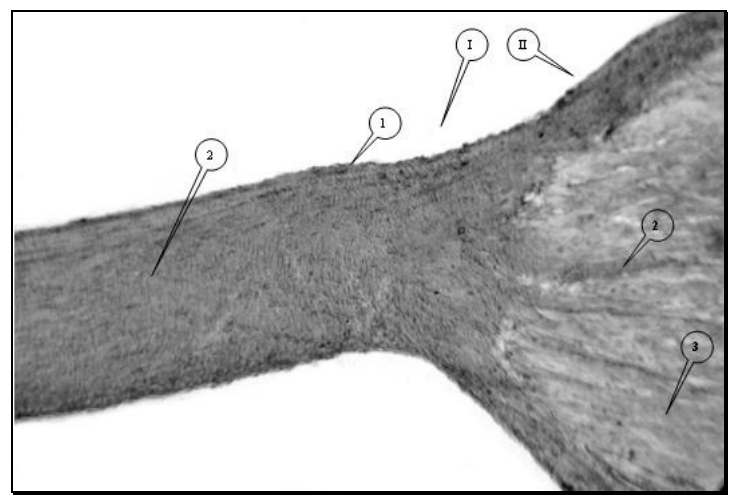

Figure 2. Longitudinal section normally located tendon strings mitral valve (place of attachment to the mastoid muscle. $\times 40$. Staining by Van Hizona. I - tendon string, II - mastoid muscle, 1 - endothelium, 2 - bundles of collagen fibers, 3 - cardiomyocytes.

In place of attachment to the leaf valve collagen fibers getting into the ventricular surface of the leaf, diverged in different directions and reach the fibrous layer of the leaf.

Variability of the structure of valvular heart disease, qualitative and quantitative deviations from the "standard" settings often caused by abnormal development of connective tissue, which is confirmed by the study of APC.

Unlike true TS anomalous string (AS) is not fixed to the valve leaflets, and the ventricular walls. We explored nine of hearts, which were present in the cells ARS left ventricle. If the studies are localized between these intracardiac entities: the posterior-medial of MM and left ventricular wall and interventricular membrane, anterior-lateral and interventricular MM membrane, mastoid muscle; wall of the left ventricle and interventricular membrane; wall of the left ventricle. Sometimes ARS had membranous structure at the ends and connected to three or more lesions. Transverse we considered AU, situated within one of ventricle. Diagonal AC was the place of attachment in the neighboring departments. Longitudinal AU believed that that stretched from the apical to basal division, often parallel to the interventricular septum.

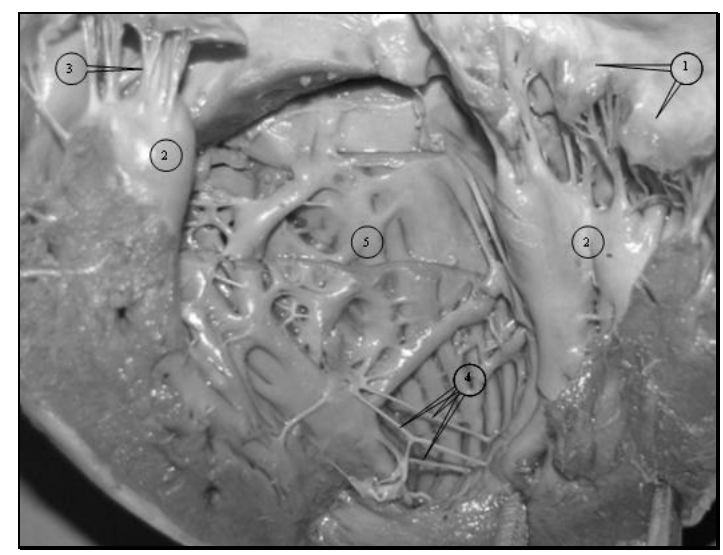

Figure 3. The cavity of the left ventricle : mitral valve normally located TS and abnormally located in the apical string section. 1 - valve valve , 2 - mastoid muscle , 3 - tendon strings, 4 - abnormally positioned string , 5 - left ventricular cavity

They were mostly represented by single strands. But sometimes multiple abnormal string in a cell of the heart is revealed, it is often located in the apical section (Fig. 3). We found TS with transverse most frequently. 
Researching optical study sections (using techniques Veyhert Van Hizona and Slinchenko ) all ARS that we found weer these patterns and morphological characteristics of their microscopic structure. The surface of all the speakers was also covered with endocardium, consisting of endothelial surface layer and deeper layer of elastic fibers located. In many cases, the AS for microscopic examination had traumatic injury, local and widespread fibrosis. AU skeleton was dense connective tissue, such as tightly packed, sent straight helical bundles of thick collagen fibers. Sometimes observed disorganization of collagen fibers without tissues and fragmentation of beams. In most cases met cardiac muscle cells, sometimes leading. If properly placed SS muscle cells were more prevalent in places discharge from the CM, the AS muscle cells had different localization. Value of connective tissue to muscle component different: some speakers dominated components of connective tissue such as collagen fibers in other cardiomyocytes. Cardiomyocytes intertwined with each other without forming a clear framework, sometimes localized islands, often accompanied by blood vessels. In some cases, muscle cells are only in the place of attachment, sometimes stretching along the string. Even sometimes seemed as if the string is divided in half lengthwise, one half had a connective tissue structure, formed by another muscle component (Fig. 4).

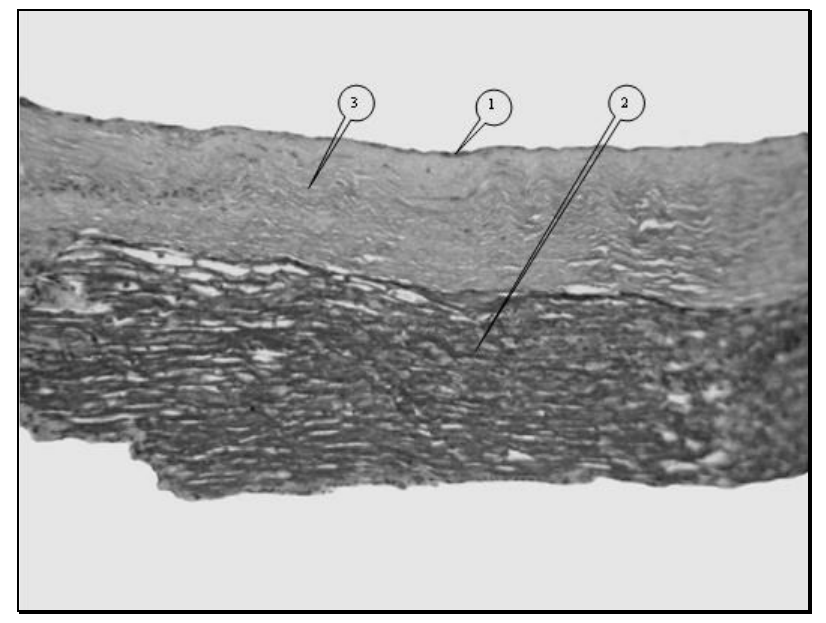

Figure 4. Longitudinal section abnormally located strings left ventricle. $\times 40$. Staining method Slinchenko. 1 - endothelium, 2 - heart muscle cells, 3 - bundles of collagen fibers

In most cases the contractile cardiomyocytes were observed, but also the key are identified, located in the inner layers of strings and were wider and shorter compared to contractility (Fig. 5). Abnormally strings in most studies were fibromuscular type.

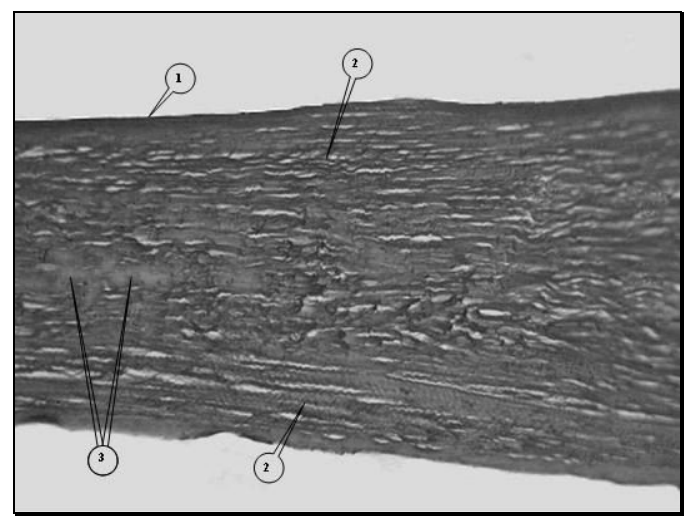

Figure 5. Longitudinal section abnormally located strings mitral valve leading to the presence of cardiomyocytes (Purkinje cells) $\times 40$. Staining method Veyherta Van Hizona. 1 - endothelium, 2 - bundles of collagen fibers, 3 - leading cardiac cells (Purkinje cells) 
Available in AC conduction system cells at specific topographical position of the trigger and trigger factors (emotional stress, physical overload, intoxication, etc.) can cause cardiac arrhythmias and functioning extra, abnormal ways of pulse on $\mathrm{AC}^{1-2}$.

Thus, histological examination of ARS needs even further morphological studies of tissue and cell structure as their relationship to cardiovascular disease is indisputable. This knowledge will help in the prediction and prevention of complications such as rupture strings arrhythmic syndrome, thrombosis of vessels and others arising in patients with ARS.

The use of lasers polarimetry in medical research is based on the use of a wide range of phenomena associated with different effects of light interaction with biological objects ${ }^{3-6}$. Highly accurate, informative, which makes no other method are the factors which actively support the development of laser diagnostic pathology biological tissues ${ }^{7-12}$.

\section{EXPERIMENTAL RESULTS AND DISCUSSION}

For the conduction of such investigations a new installation was created which optical scheme is shown in Fig. 6.

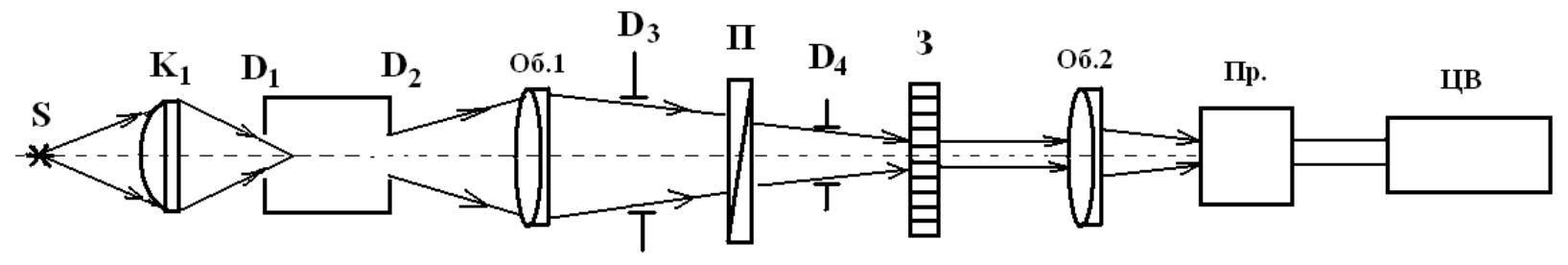

Figure 6. Structure functional scheme of the installation

Here $S$ is a stabilized source of radiation; $K_{1}$ is a lighted condenser; $D_{1} i D_{2}$ is an entrance and exit slot of the monochromator MUM; O.1 i O.2 are objective glasses; $\mathrm{D}_{3}$ i $\mathrm{D}_{4}$ are diaphragms; $\mathrm{P}$ is a polarizer, Nicol prism; 3 is an investigated sample; $\mathrm{D}$ is a radiation detector: в in the area $\lambda=330-600 \mathrm{~nm}$ FEU-39, in the area $\lambda=600-750 \mathrm{~nm}$ the silicon photodiode FD-24K; DV is a digital voltmeter.

The sample 3 is fastened in the limb and can turn round in the beam from $0 \%$ to $360 \%$ relative to the area of radiation polarization after the polarizer. It gives the possibility to determine the magnitude of the minimum transmission (at the predominant orientation of the sample structure elements parallel to the area of polarization) - $\tau_{0}$ and maximum transmission (at the predominant orientation of the sample structure elements perpendicularly to the area of polarization) - $\tau_{90}$ for each given wave length $\lambda$ by the monochromator. In order to obtain the absolute factor values of the transmission in the direct beam the investigated sample is taken out of the beam zone - the digital voltmeter registers the magnitude of the signal norm $\mathrm{I}_{0}$.

Analytical description of the processes of transformation parameters of optical radiation biological tissues (BT) of different types based on the use of Mueller matrix for optically uniaxial structures ${ }^{13}$

$$
\{Q\}=\left\|\begin{array}{cccc}
1 ; & 0 ; & 0 ; & 0 ; \\
0 ; & \left(\sin ^{2} \frac{\delta}{2} \cdot \cos 2 \rho+\cos ^{2} \frac{\delta}{2}\right) ; & \left(0,5 \sin 4 \rho \sin ^{2} \frac{\delta}{2}\right) ; & (-\sin 2 \rho \sin \delta) ; \\
0 ; & \left(0,5 \sin 4 \rho \sin ^{2} \frac{\delta}{2}\right) ; & \left(-\sin ^{2} \frac{\delta}{2} \cdot \cos 2 \rho+\cos ^{2} \frac{\delta}{2}\right) ; & (\cos 2 \rho \sin \delta) ; \\
0 ; & (\sin 2 \rho \sin \delta) ; & (-\cos 2 \rho \sin \delta) ; & \left(2 \cos ^{2} \frac{\delta}{2}-1\right) ;
\end{array}\right\|
$$

Here $\rho$ is an optical orientation axis direction is determined conclusion fibrils, $\delta$ is the value of the phase shift that brought them matter between orthogonal components of the amplitude of the laser wave.

In the framework of this model ${ }^{14-16}$ was able to resolve the problem directly and explain the mechanisms of polarization structure of object fields of biological tissue types. On this basis, if a single scattering established relationship between the values of azimuth, ellipticity of polarization of light vibrations images of biological tissue and directions laying optical axes fibrils.

Next, there are given the results of comparative statistics, correlation and fractal study population Mueller-matrix images (MMI) of healthy and abnormal (early forms that are not diagnosed by histological methods) BT normal and abnormally located tendon strings left ventricle of the human heart.

Based on modeling of polarization properties of the matrix BT statistical correlations between moments of 1-4 orders of coordinate distributions of matrix elements with geometric and anisotropic structure of architectonic nets was analyzed. 

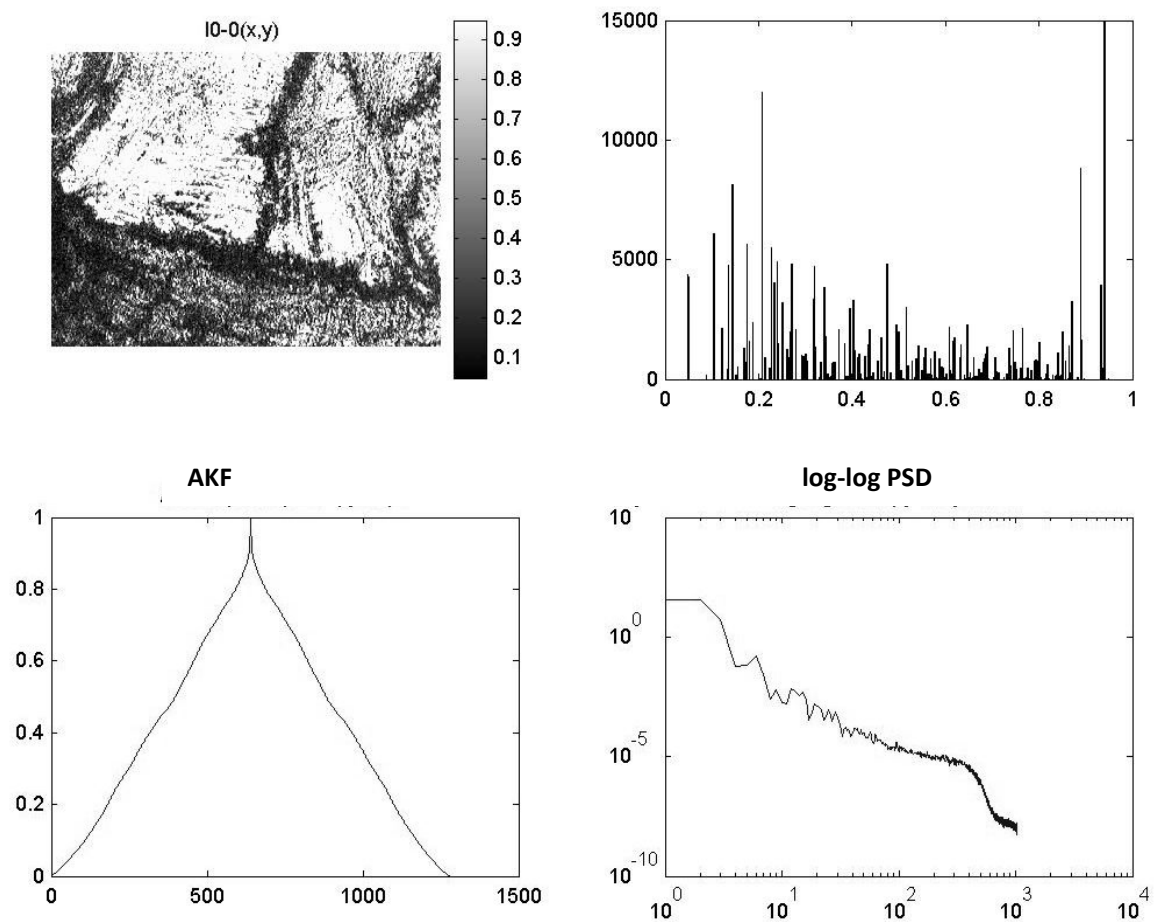

Fig.7. Image abnormally located tendon strings left ventricular human heart and its statistical, correlation and spectral analysis.
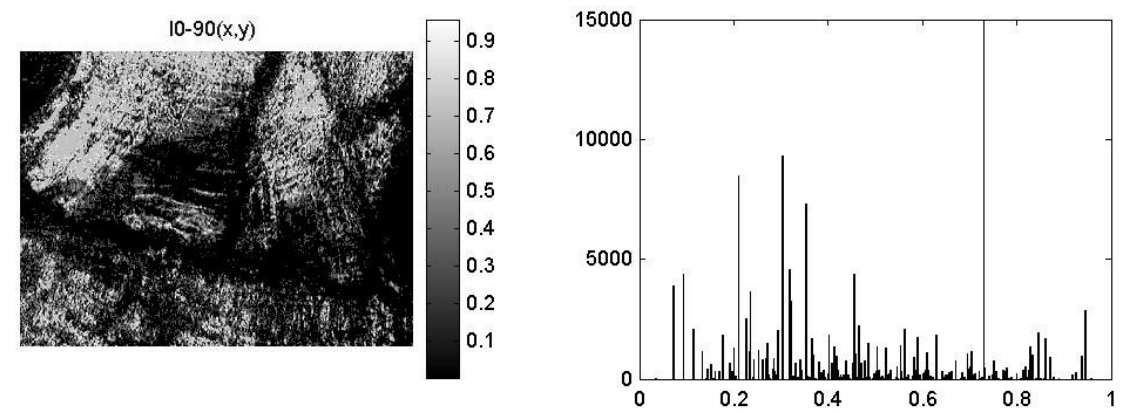

AKF
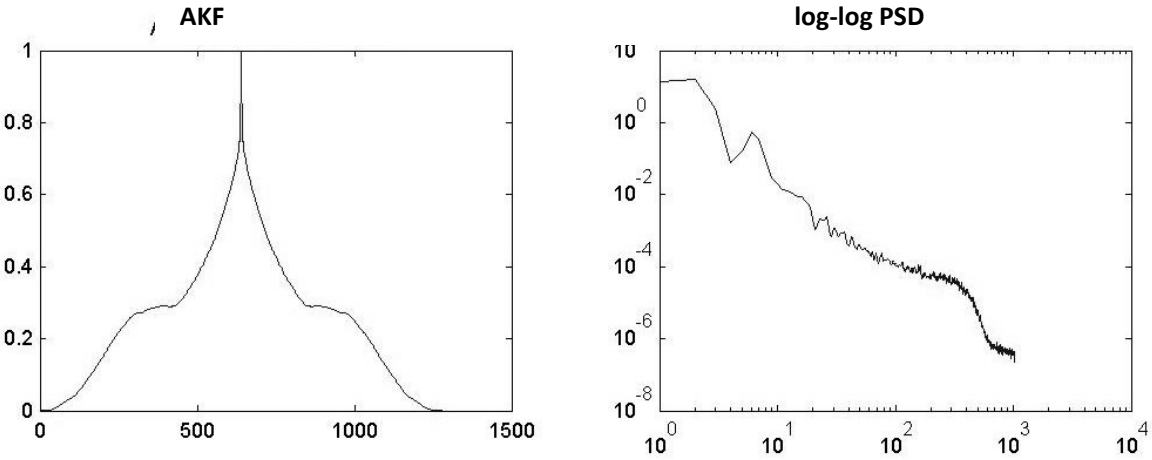

Fig.8. Polarization image abnormally located tendon strings left ventricle of man and its statistical, correlation and spectral analysis.

Proc. of SPIE Vol. 9066 90661Q-6 
In this work the power spectra together Mueller- matrix images of BT was studied. We found that the distribution of coordinate "orientation" matrix elements physiologically normal BT characterized by a fractal structure. The range of power distribution "phase" stochastic matrix element. Established that the pathologically altered architectonics BT coordinate orientation distribution stochastic matrix elements. Stochastic Structure of the coordinate distribution is transformed into a statistical element. Based on the data obtained the degree of self-similarity coordinate distributions of Mueller matrix elements formulated the principles of fractal differentiation of physiological state.

As the basic analytical expression for calculating the spectral autocorrelation functions

$$
G_{x x}\left(M_{i k}\left(\lambda_{1}, \lambda_{2}\right)\right) \text { of }
$$

statistical distributions $M_{i k}\left(Z_{\rho}^{(2)}, \Delta_{1}\left(\lambda_{1}\right)\right)$ and $M_{i k}\left(Z_{\rho}^{(2)}, \Delta_{2}\left(\lambda_{2}\right)\right)$ matrix elements we have used the expression given in ${ }^{6}$

$$
G_{x x}(\Delta x)=\lim _{x \rightarrow 0} \frac{1}{X_{0}} \int_{0}^{X_{0}}\left[M_{i k}(x)\right]\left[M_{i k}(x-\Delta x)\right] d x
$$

This "step" $\Delta x$, which changes the coordinates $(x)$ of the statistical distribution of the studied element $M_{i k}$ Mueller matrix of biological tissue.

Power spectrum $P\left(G_{x x}\right)$ calculated by the following expression:

$$
P\left(G_{x x}\right)=\int_{1}^{x_{0}} G_{x x}(\Delta x) \cos 2 \pi v_{x} x d x
$$

here $v_{x}$ - the spatial frequency that is inversely proportional to the size $L$ of the structural birefringent elements.

Comparative analysis of the correlation structure of statistical distributions "diagonal" and "diagonal" elements of the Mueller matrix of biological tissue birefringent crystals found:

- half-width of the autocorrelation function $G_{x x}\left(M_{22}\right)$ (Fig. 7-8) for two wavelengths of laser radiation tends to reduce the bias in the "blue" part of the spectrum $X^{*}\left(M_{22}\left(\lambda_{1}\right)\right) / X^{*}\left(M_{22}\left(\lambda_{2}\right)\right) \approx 3$;

- half-width of the autocorrelation functions $G_{x x}\left(M_{34}\right)$ "diagonal" element Mueller matrix (Figure 7) has a similar, but more marked tendency $X^{*}\left(M_{34}\left(\lambda_{1}\right)\right) / X^{*}\left(M_{34}\left(\lambda_{2}\right)\right) \approx 5$.

The data on the correlation structure of the statistical distributions of the matrix elements $M_{i k}\left(\lambda_{1}, \lambda_{2}\right)$ are in good agreement with the model trend analysis of spectral changes in the values of half-width dependencies $G_{x x}\left(M_{i k}\right)$.

Here are the data structures polarization abnormally located tendon strings left ventricle of the heart of man - maps azimuth, ellipticity of the polarization of its structure (Fig. 9-10).

Thus, the results of experimental research and statistical correlation structure of the spectral dependence of the twodimensional Mueller matrix elements and phase shifts of histological sections of different morphological structure and physiological state. The established relationship between the distribution of orientations of the optical axes birefringent miozyn fibrils with a set of statistical moments that characterize the distributions of Mueller matrix elements in different spectral ranges and half-width corresponding autocorrelation functions. 
Azimus 0

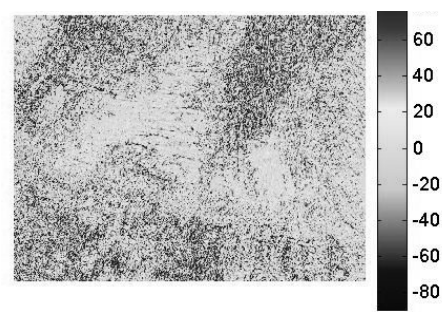

Azimus 45

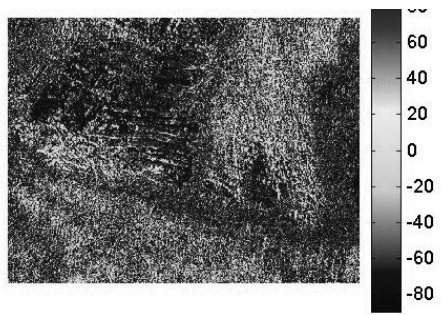

Azimus 90

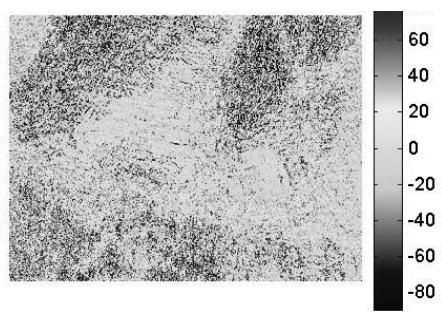

Azimus R

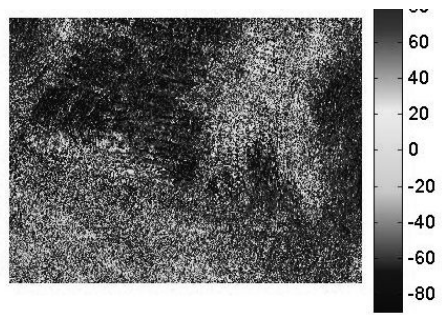

Figure 9. Polarization structure of abnormally located tendon strings left ventricle of the heart of man - maps azimuth of polarization.
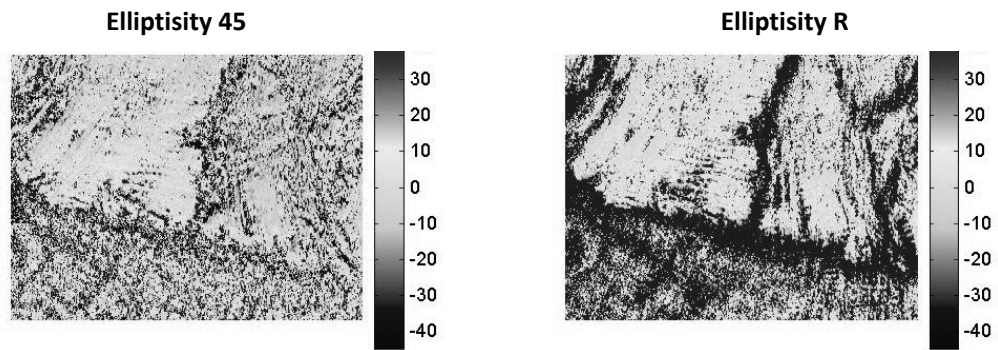

Figure 10. Polarization structure of abnormally located tendon strings left ventricle of the heart of man - maps ellipticity of polarization. 


\section{CONCLUSIONS}

The morphological peculiarities of TS mitral valve of the heart of man in normal and abnormal spaced strings of the left ventricle and the study of their structural features depending on the location was studied.

Results of statistical dependency and the correlation structure of two-dimensional Mueller matrix elements in different spectral ranges of laser radiation on changes in the distribution of orientations of the optical axes and birefringence of protein crystals biological tissues revealed. Diagnostic sensitivity to changes in optical anisotropic component of biological objects of statistical (statistical moments of the 1st - 4th order) and correlation (half-width of the autocorrelation function and the variance of the power spectra) parameters was founded.

\section{Acknowledgments}

This work was supported by grants F53/103-2013 from the Fundamental Researches State Fund of Ukraine and \#13-02-90468Ukr_f_a from the Russian Foundation for Basic Research.

\section{REFERENCES}

[1] Glesby, M.J., "Assotiation of mitral valve prolapse and systemic abnormalities of connective tissue. A phenotypic continuum," J. Amer. Med. Ass., 262, 523-528 (1989).

[2] Millingston-Sanders, C., Meir, A., Lavrence, L., Stolinski, C., "Stucture of chordae tendineae in the left ventricle of the human heart," J. Anat., 192, 573-581 (1998).

[3] Cowin, S.C., "How is a tissue built?," J. Biomed. Eng., 122, 553-568 (2000).

[4] [Handbook of Coherent-Domain Optical Methods. Biomedical Diagnostics, Environmental and Material Science] ed. V.Tuchin. - Kluwer Academic Publishers (2004).

[5] Angelsky, O.V., Mokhun, A.I., Mokhun, I.I, Soskin, M.S. "The relationship between topological characteristics of component vortices and polarization singularities," Optics communications 207 (1), 57-65 (2002).

[6] Gerrard, J.M. [Introduction to matrix methods in optics], New York.: A Wiley-Interscience Publ. (1975).

[7] Angelsky, O.V., Burkovets, D.N., Kovalchuk, A.V., Hanson, S.G. "Fractal description of rough surfaces," Applied optics 41 (22), 4620-4629 (2002).

[8] Angelsky, O.V., Burkovets, D.N., Maksimyak, P.P., Hanson, S.G. "Applicability of the singular-optics concept for diagnostics of random and fractal rough surfaces," Applied optics 42 (22), 4529-4540 (2003).

[9] Angelsky, O.V., Ushenko, A.G., Burkovets, D.N., Ushenko, Y.A. "Polarization visualization and selection of biotissue image two-layer scattering medium," Journal of biomedical optics, 10 (1), 14010 (2005).

[10] Angelsky, O.V., Ushenko, A.G., Ushenko, Y.A., Ushenko, Y.G. "Polarization singularities of the object field of skin surface," Journal of Physics D: Applied Physics 39 (16), 3547 (2006).

[11] Angelsky, O.V., Yermolenko, S.B., Prydij, O.G., Ushenko, A.G., Ushenko, Ye.G. "Polarization-interference structure of speckle fields of the rough skin surface," J. Holography Speckle 3, 27-34 (2006).

[12] Angelsky, O.V., Yermolenko, S.B., Zenkova, C.Yu., Angelskaya, A. O., "Polarization manifestations of correlation (intrinsic coherence) of optical fields," Appl. Opt., 47, 5492-5499 (2008).

[13] Yermolenko, S.B., Prydij, A.G., Vladychenko, K.V. "Polarimetry of multi-layer biological tissue," SPIE Proc., 7008, 70082D-1 - 70082D-7 (2008).

[14] Yermolenko, S.B., Ivashko, P.V., Prydij,A.G., Gruia, I. "Spectral and biochemical methods for identification of cellular changes and malignant tissues," Optoelectronics and advanced materials - rapid comunications, 4 (4), 523-526 (2010).

[15] Angelsky O.V., Polyanskii P.V., Felde C.V. "The emerging field of correlation optics," Optics and Photonics News, 23(4), 25-29 (2012)

[16] Angelskaya, A.O., Gruia, I., Yermolenko, S.B., Ivashko, P.V., Gruia, M.I., "Manifestations of linear dichroism changes in cancer biotissues," Romanian Reports in Physics, 65 (3) 1052-1062, (2013). 